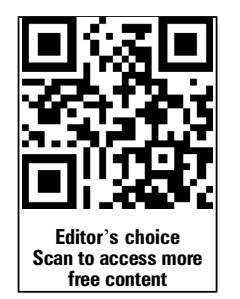

- To read the full version of this paper, please visit the journal online (http://dx.doi. org/10.1136/bjsports-2013092690).

${ }^{1}$ Department of Exercise and Sport Sciences, School of Health Sciences and Human Performance, Ithaca College, Ithaca New York, USA ${ }^{2}$ Department of Kinesiology, Curry School of Education, University of Virginia,

Charlottesville, Virginia, USA ${ }^{3}$ Department of Biology, University of Utah, Salt Lake City, Utah, USA

${ }^{4}$ Department of Physical Medicine and Rehabilitation, Spaulding National Running Center, Harvard Medical School, Cambridge, Massachusetts, USA

\section{Correspondence to} Dr Patrick O McKeon, Department of Exercise and Sport Science, School of Health Sciences and Human Performance, Ithaca College Hill Center, Room G66 953

S. Danby Rd, Ithaca, NY

14850, USA;

pmckeon@ithaca.edu

Accepted 27 February 2014

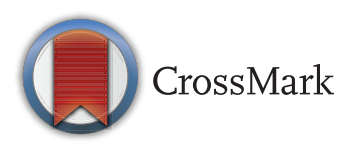

To cite: McKeon $\mathrm{PO}$, Hertel J, Bramble D, et al. $\mathrm{Br} J$ Sports Med 2015;49:290.

\title{
The foot core system: a new paradigm for understanding intrinsic foot muscle function
}

\author{
Patrick O McKeon, ${ }^{1}$ Jay Hertel, ${ }^{2}$ Dennis Bramble, ${ }^{3}$ Irene Davis $^{4}$
}

\section{ABSTRACT}

The foot is a complex structure with many articulations and multiple degrees of freedom that play an important role in static posture and dynamic activities. The evolutionary development of the arch of the foot was coincident with the greater demands placed on the foot as humans began to run. The movement and stability of the arch is controlled by intrinsic and extrinsic muscles. However, the intrinsic muscles are largely ignored by clinicians and researchers. As such, these muscles are seldom addressed in rehabilitation programmes. Interventions for foot-related problems are more often directed at externally supporting the foot rather than training these muscles to function as they are designed. In this paper, we propose a novel paradigm for

understanding the function of the foot. We begin with an overview of the evolution of the human foot with a focus on the development of the arch. This is followed by a description of the foot intrinsic muscles and their relationship to the extrinsic muscles. We draw the parallels between the small muscles of the trunk region that make up the lumbopelvic core and the intrinsic foot muscles, introducing the concept of the foot core. We then integrate the concept of the foot core into the assessment and treatment of the foot. Finally, we call for an increased awareness of the importance of the foot core stability to normal foot and lower extremity function.

The human foot is a very complex structure, which allows it to serve many diverse functions. During standing, it provides our base of support. During gait, the foot must be stable at foot-strike and push-off. However, during mid-support, the foot must become a mobile adaptor and attenuate loads. It also possesses spring-like characteristics, storing and releasing elastic energy with each foot-strike. This is accomplished through the deformation of the arch, which is controlled by intrinsic and extrinsic foot muscles. There is evolutionary evidence that the foot arch architecture and musculature developed in response to the increased demands of load carriage and running. The stability of this arch, which we proposed to be the central 'core' of the foot, is requisite to normal foot function.

\section{THE RELEVANCE OF CORE STABILITY TO THE FOOT}

Core stability has received much attention in the clinical and athletic arenas. Interest has primarily been focused on the role of lumbopelvic-hip stability in normal lower extremity movement patterns. ${ }^{1}$ The muscular system of the lumbopelvic hip complex, or core, has been described as consisting of local stabilisers such as the multifidus and transverse abdominis, and global movers such as latissimus dorsi. ${ }^{2}$ The local stabilisers have small cross-sectional areas and small moment arms. Therefore, they do not produce large rotational moments at the respective joints that they cross. However, they do act to increase intersegmental stability. Proper function of local stabilisers provides a stable base on which the primary movers of the trunk, those with larger cross-sectional areas and moment arms, can act to cause gross motion. When core muscles are weak or are not recruited appropriately, the proximal foundation becomes unstable and malaligned, and abnormal movement patterns of the trunk and lower extremity ensue. ${ }^{3}$ This can lead to a variety of overuse lower extremity injuries. ${ }^{4-7}$

We propose that the concept of core stability may also be extended to the arch of the foot. The arch is controlled with both local stabilisers and global movers of the foot, similar to the lumbopelvic core. The local stabilisers are the four layers of plantar intrinsic muscles that originate and insert on the foot. These muscles generally have small moment arms, small cross-sectional areas and serve primarily to stabilise the arches. The global movers are the muscles that originate in the lower leg, cross the ankle and insert on the foot. These muscles have larger cross-sectional areas, larger moment arms, are prime movers of the foot, and also provide some stability to the arch. With each footstep, the four layers of intrinsic muscles act to control the degree and velocity of arch deformation. When they are not functioning properly, the foundation becomes unstable and malaligned; and abnormal movement of the foot ensues. This may manifest in foot-related problems. Plantar fasciitis is one of the most common overuse injuries of the foot. It is recognised as a repetitive strain injury from excessive deformation of the arch. ${ }^{8}$ The importance of the arch musculature in this prevalent foot injury is currently underappreciated. This is underscored by recent articles describing clinical evidence and guidelines for plantar fasciitis, ${ }^{9}$ as well as posterior tibial tendon dysfunction, ${ }^{10}$ medial tibial stress syndrome ${ }^{11}$ and chronic lower leg pain ${ }^{12}$ that have no mention of foot strengthening as a component of the interventions.

Therefore, our purpose was to propose a foot core system paradigm by (1) describing the evolution of the human arch for locomotion, (2) delineating the subsystems of the foot core, (3) reviewing assessment and treatment of the foot integrating the concepts of foot core stability and (4) finally discussing future research directions. Our overall goal was to propose a new paradigm by which to view foot function, assessment and treatment. 


\section{THE ORIGIN OF THE HUMAN ARCH}

The human foot has evolved from one similar to that of African apes, where it serves in both arboreal and terrestrial locomotion. ${ }^{13}$ The transition from ape-like to human-like foot structure reflects a shift to locomotor habits dominated by terrestrial bipedality. When walking bipedally, the gait of chimps is compromised by the absence of structural specialisations that permit the human foot to operate as a compact, rigid lever system during the latter half of stance. These include (1) an enlarged and permanently adducted hallux, (2) shortened lateral digits, (3) compaction and realignment of the tarsal bones to help prevent the 'mid-tarsal break' observed in the foot of apes ${ }^{14}$ and (4) the addition of a welldefined medial longitudinal arch defended by strong plantar tensile elements. The condition of the foot arches, absent in apes, remains controversial. A transverse arch was likely present, ${ }^{15}$ but the crucial medial longitudinal arch was absent or weakly expressed, ${ }^{16}$ implying a poorly defined plantar aponeurosis and hence greater reliance on muscular effort to resist forces acting on the toes during late stance. In contrast, even the earliest members of the genus Homo for which there is adequate evidence (eg, early Homo erectus) possessed an essentially modern foot structure, including a well-defined medial longitudinal arch. ${ }^{17}$

The modern human body (ie, Homo), especially in musculoskeletal design, reflects the mechanical demands of endurance running. ${ }^{18}$ That habitual bipedalism was practiced for several million years by Australopithecus without the hallmark features of the human foot also suggests that such traits arose in the context of a new and more demanding locomotor behaviour. A key distinction between walking and running is the central importance of 'leg springs' in running but not walking. ${ }^{19}$ These springs include a pronounced Achilles tendon and the plantar aponeurosis and spring ligaments on the inferior aspect of the foot. All are absent in apes and were either lacking or minimally developed in Australopithecus. Running also subjects the digits to much larger extension forces during late stance and toe-off phases than does walking; a strong plantar aponeurosis offers substantial passive resistance to these loads. Additionally, mid-stance flattening of the longitudinal arch when running both cushions foot impact and stores recoverable strain energy in the stretched elastic tissues, ${ }^{20}$ but unlike most quadrupedal mammals specialised for running, humans retain considerable intrinsic foot musculature. These same muscles are reduced and sometimes completely lost in quadrupedal runners, making internal stabilisation of the foot mostly passive. Human runners are unique in needing to control balance during single leg support and for this reason (unlike quadrupeds) require a foot that is reasonably mobile, able to accommodate uneven substrates, and actively controlled. Electromyography (EMG) studies show that plantar intrinsic foot muscle activity is most consistent among participants during running and least during walking. ${ }^{21}$ While more variable, the intrinsic foot muscles are routinely active in late stance of walking and may have a significant role in controlling load distribution under the foot as well as augmenting the flexor function of the medial longitudinal arch, especially at higher speeds. ${ }^{21} 22$ Although often showing minimal activity in simple stance, the intrinsic foot muscles are more strongly recruited when additional loads are added to the participant. ${ }^{23}$ Frequent long-distance burden carrying may explain the evolutionary transformation from Australopithecus-like to Homo-like body proportions $^{24}$ and might also help account for relatively robust intrinsic foot muscle development in the human foot.

\section{THE FOOT CORE SYSTEM}

The theoretical basis of lumbopelvic-hip core stability is rooted in the functional interdependence of the passive, active and neural subsystems controlling spinal motion and stability originally proposed by Panjabi. ${ }^{25}$ The passive subsystem consists of the bony and articular structures, while the active subsystem consists of the muscles and tendons attaching to and acting on the spine. The neural subsystem consists of sensory receptors in the joint capsules, ligaments, muscles and tendons surrounding the spine. The passive subsystem provides for a balance between mobility and stability of the vertebral column. The active subsystem consists of two functional muscular components: the local stabilisers and the global movers. ${ }^{2}$ The local stabilisers consist of both the short, intersegmental muscles that primarily originate and insert on the spinal column and have short moment arms and act to increase intersegmental dynamic stability. Proper function of local stabilisers provides a stable base on which the primary movers of the trunk can act to cause gross motion. The global movers cross multiple vertebral segments, have attachments on the pelvis and thorax, and can exert longer moment arms to move the trunk and extremities. These include the more superficial erector spinae as well as the internal and external oblique and rectus abdominus muscles. The neural subsystem monitors spinal motion and forces and sends afferent signals to the central nervous system. If those afferent signals exceed a given threshold, efferent signals are sent from the central nervous system to the appropriate muscles to alter spinal motion and forces.

Hodges $^{26}$ further delineated the strategies of lumbopelvic core stability into 'control' and 'capacity' components. The control strategy aims to restore coordination of the muscles acting on the lumbopelvic core while the capacity strategy aims to provide adequate muscle strength and endurance to prevent the spine from being mechanically unstable under varying loads. Ultimately, the control and capacity strategies complement each other in providing for a stable lumbopelvic core and these same principles may be applied to the foot core system. The application of lumbopelvic core stability concepts to the foot are illustrated in figure 1 . These concepts as they relate to the ankle and foot were first proposed by $\mathrm{Jam}^{27}$ and we further expand on their application to the foot core. The description of each of subsystems follows.

\section{Passive subsystem of the foot core}

The passive subsystem of the foot core consists of the bones, ligaments and joint capsules that maintain the various arches of the foot. The functional configuration of the bony anatomy of the foot results in four distinct arches which include the medial and lateral longitudinal arches as well as the anterior and posterior transverse metatarsal arches. ${ }^{28}$ While often viewed as separate structures, McKenzie ${ }^{29}$ proposed that these arches coalesce into a functional half dome responsible for flexibly adapting to load changes during dynamic activities (see figure 2). This half dome has been thought to be predominantly supported by passive structures including the plantar aponeurosis (see figure $3 \mathrm{~A}$ ) and plantar ligaments (see figure 4), however local dynamic support is also thought to be provided from the intrinsic foot muscles in the active subsystems and indirectly by the contractions of the extrinsic foot muscles. ${ }^{30}$

\section{Active subsystem of the foot core}

The active subsystem consists of the muscles and tendons that attach on the foot. The local stabilisers of the foot are the plantar intrinsic muscles that both originate and insert on the foot, whereas the global movers are the extrinsic muscles that originate in the lower leg, cross the ankle and insert on the foot (see figure 5). While there are intrinsic muscles on both the dorsal and plantar aspects, the plantar intrinsic muscles are most 
Figure 1 The foot core system. The neural, active and passive subsystems interact to produce the foot core system which provides stability and flexibility to cope with changing foot demands.

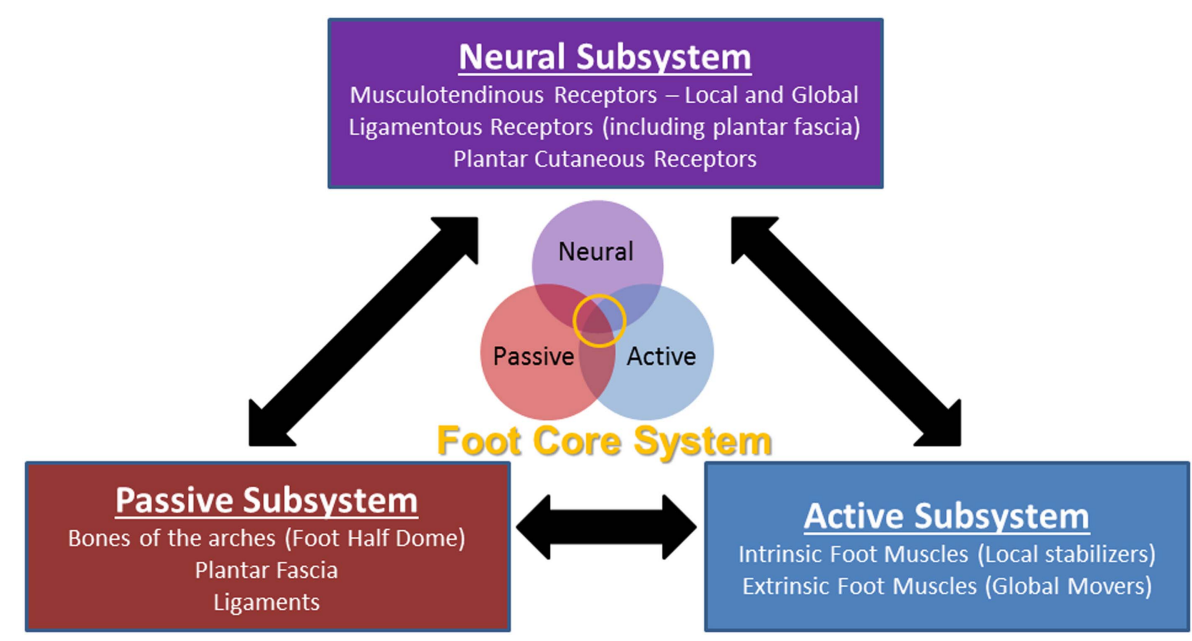

commonly described due to their functional link with the longitudinal and transverse arches of the foot half dome. ${ }^{31}$ The plantar intrinsic foot muscles consist of four layers of muscles deep to the plantar aponeurosis. The first two layers have muscle configurations which align with the medial and lateral longitudinal arches of the foot whereas the deeper layers configure more so with the anterior and posterior transverse arches (see figure 6A-E). See online supplementary appendix for a full description of the anatomical and biomechanical contributions of the intrinsic foot muscles. By examining the synergistic relationships among these muscles to the relevant bony anatomy and foot dome configuration, their functional role may be elucidated. Soysa $e t a l^{31}$ summarised the functional qualities of the intrinsic foot muscles to include being supportive of the foot arches, ${ }^{32-35}$ activity and load dependent, ${ }^{36} 37$ synergistic $^{38}$ and modulating. ${ }^{28}$ See table 1 for the evidence-based descriptions of these functional qualities.

The extrinsic foot muscles function as the global movers of the foot core to generate foot motion via their long tendons and modulate structures within the passive subsystem. For example, the Achilles tendon from the triceps surae modulates the tension of the plantar aponeurosis based on their common connection to the calcaneus. As triceps surae tension increases, so does the tension on the plantar fascia ${ }^{39}$ (see figure 3B). This is critically important for key events in foot behaviour such as transitioning from a supple to a rigid body during gait. The orientations of the extrinsic foot muscle tendons clearly illustrate their ability to provide dynamic support and control of both the longitudinal and transverse components of the foot dome. These global movers provide both absorption and propulsion capabilities during dynamic activities.

\section{Neural subsystem of the foot core}

The neural subsystem consists of the sensory receptors in the plantar fascia, ligaments, joint capsules, muscles and tendons involved in the active and passive subsystems. It is well accepted that plantar sensation is a critical element to gait and balance with the contributions of the plantar cutaneous receptors the most extensively studied. ${ }^{40-44}$ The sensory contributions of the intrinsic foot muscles remain less clear. Based on the intrinsic foot muscles' anatomical and biomechanical configuration, these muscles lack mechanical advantage for producing large joint motions. Rather, their anatomical positions and alignments suggest that they are advantageously positioned to provide
Figure 2 Functional half dome proposed by McKenzie. Note the origin of the dome is considered to be the dome of the talus.
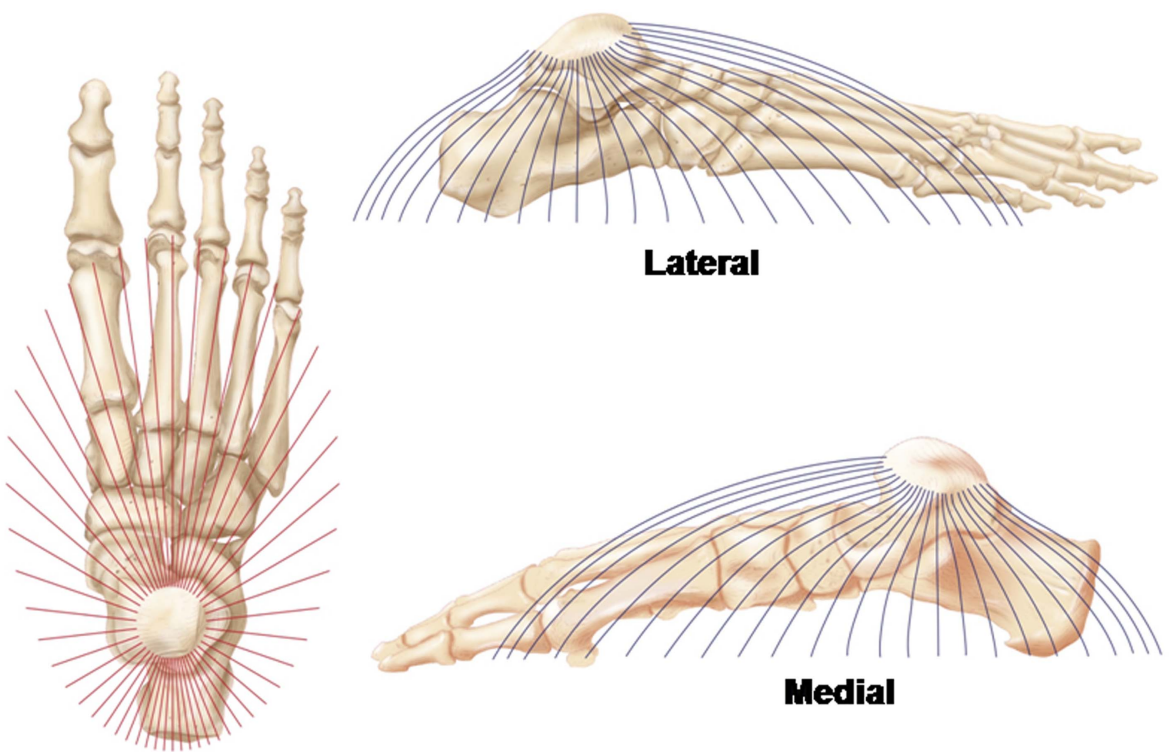

\section{Lateral}

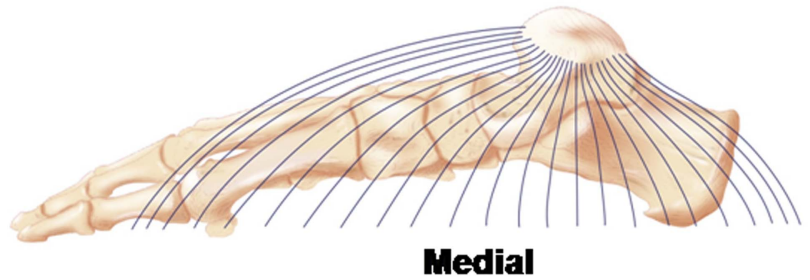

Superior 
Figure 3 (A) The plantar fascia alignment. (B) The anatomical and biomechanical relationship between the Achilles tendon and the plantar fascia is depicted. Note the fascial connection between these two structures around the calcaneus.

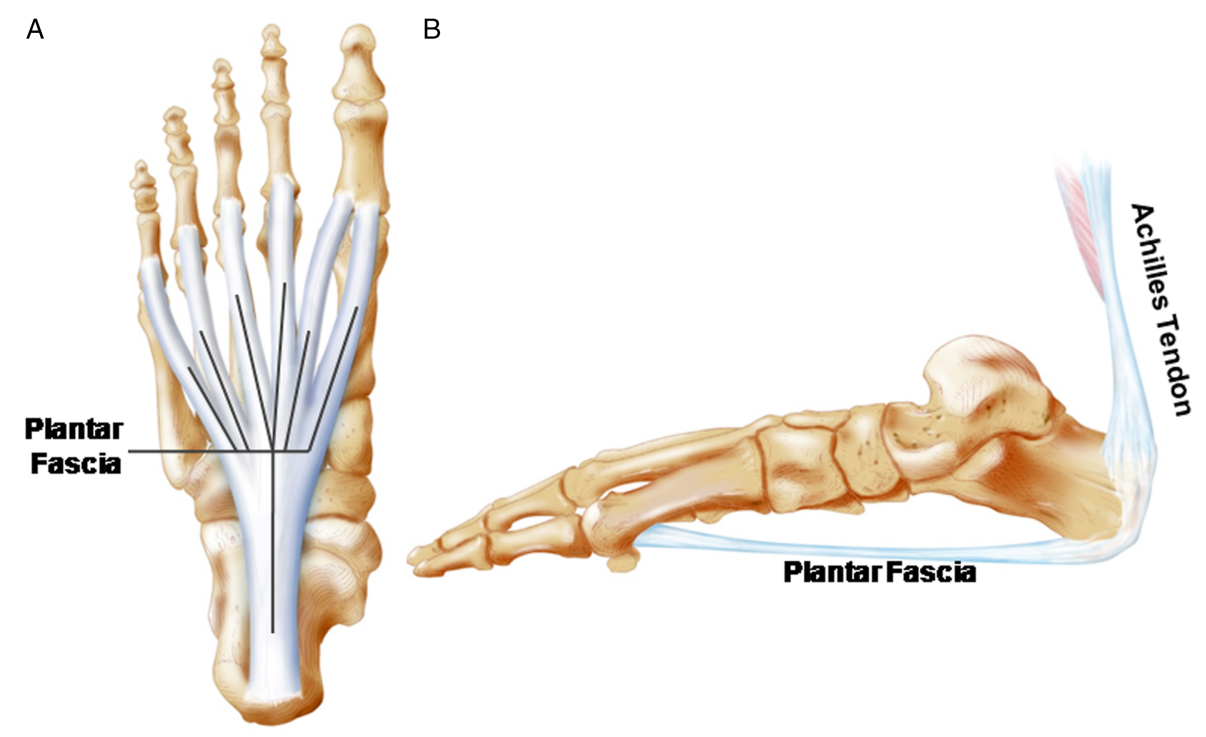

immediate sensory information, via the stretch response, about changes in the foot dome posture. In contrast to input from sensory receptors within the passive subsystem (eg, capsuloligamentous and cutaneous receptors), these sensors may be modulated through training to alter their sensitivity to foot dome deformation. ${ }^{45}$ After fatigue of the intrinsic foot muscles via repetitive isolated contractions of metatarsophalangeal joint flexion, navicular drop during standing increased significantly in

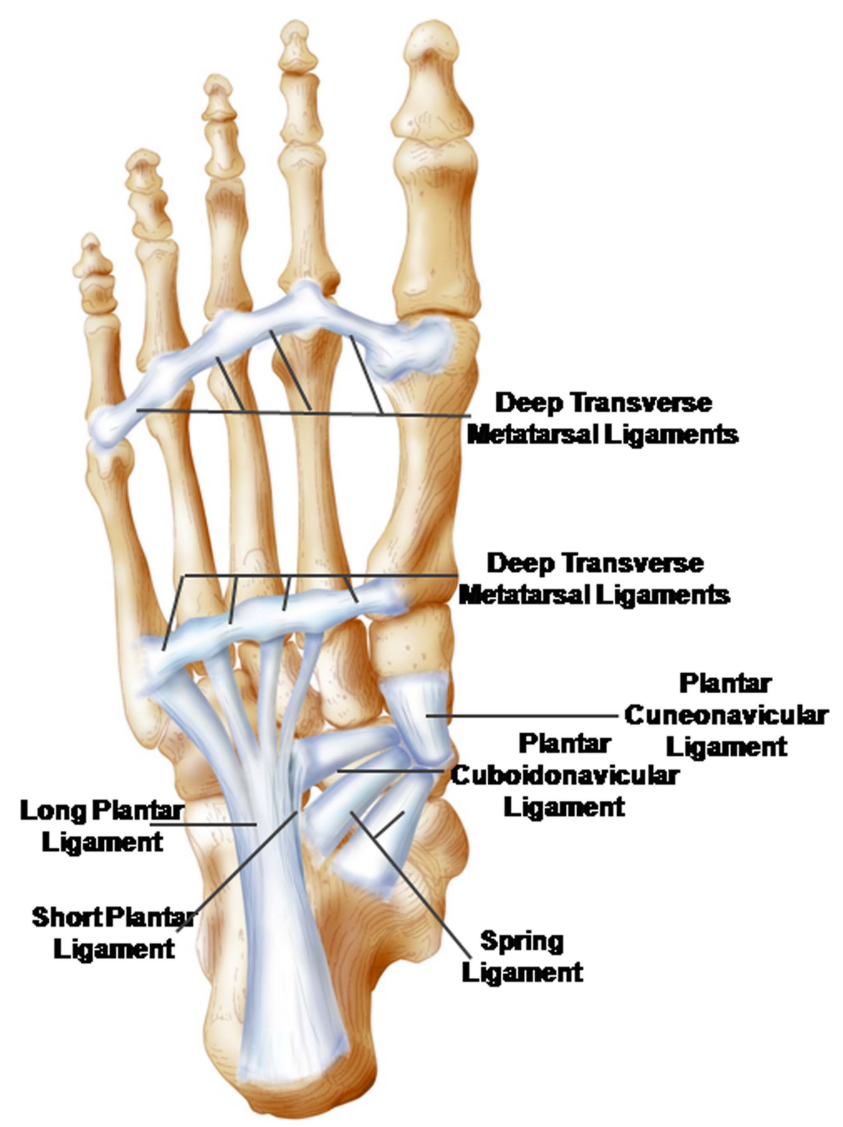

Figure 4 The predominant plantar ligaments of the foot that provide passive stability to both the longitudinal and transverse aspects of the foot. healthy participants. ${ }^{33}$ The authors concluded that the motor contributions of these muscles led to the change in foot posture, but this may be more associated with a change in sensory information. Muscular fatigue brought about by repetitive contractions has been shown to decrease joint position sense in other areas of the lower extremity. ${ }^{46}$ This may indicate that not only do the muscles provide relevant direct support to the passive subsystem via muscular contraction, but they may also provide relevant sensory information about foot dome posture in a similar fashion to the lumbopelvic muscles in relation to trunk posture. $^{47}$

\section{FOOT CORE ASSESSMENT}

Little attention has been paid to the clinical assessment of intrinsic foot muscles in the musculoskeletal injury literature apart from few specific conditions such as diabetic neuropathy ${ }^{48}$ and claw toes. ${ }^{49}$ Assessments for these conditions have largely been related to diminished toe flexion strength or atrophy of the intrinsic foot muscles. A recent systematic review concluded that there is no gold standard for assessing the function of the intrinsic foot muscles. ${ }^{31}$ Assessment techniques have been categorised as 'direct' and 'indirect' evaluations of intrinsic muscle function. ${ }^{31}$ Direct evaluations have focused on assessment of toe flexion strength while indirect evaluations include imaging techniques and EMG to estimate intrinsic foot muscle function.

Tests focusing on toe flexion strength are inherently limited by the inability to conclusively separate the contributions of the intrinsic and extrinsic toe flexor muscles. Methods of assessment have included manual muscle testing, toe grip dynamometry, pedobarography, and a pair of special tests: the paper grip and intrinsic positive tests. ${ }^{31}$ The limitation of all of these measures is their strict focus on the role of the intrinsic muscles in producing toe flexion, but ignoring their more proximal functions of supporting the arches of the foot. We assert that the latter is more important than the former is assessment of foot core function.

The intrinsic foot muscle test has been proposed as a functional assessment of a patient's ability to maintain a neutral foot posture and medial longitudinal arch height during single limb stance. ${ }^{27}$ To perform this test, the clinician sets the patient's test foot in subtalar neutral with the calcaneus and all the metatarsal heads on the ground, and asks the patient to fully extend the toes. The patient then lowers their toes to the 
Figure 5 Insertions of the extrinsic foot muscle tendons on the plantar surface of the foot. (A) The insertions of the flexor digitorum longus, flexor hallucis longus and peroneus longus are depicted. Note the longitudinal alignment of the flexor tendons as it relates to their functional contributions to longitudinal foot stability. The oblique alignment of the peroneus longus tendon and its midfoot orientation clearly supports the transverse arch. (B) The insertion of the tibialis posterior tendon is depicted with the tendons of figure $1 \mathrm{~A}$ cut away. Note the widespread insertions of the tibialis posterior tendon across the tarsals and metatarsals elucidating its functional contributions to longitudinal and transverse arch stability.
A

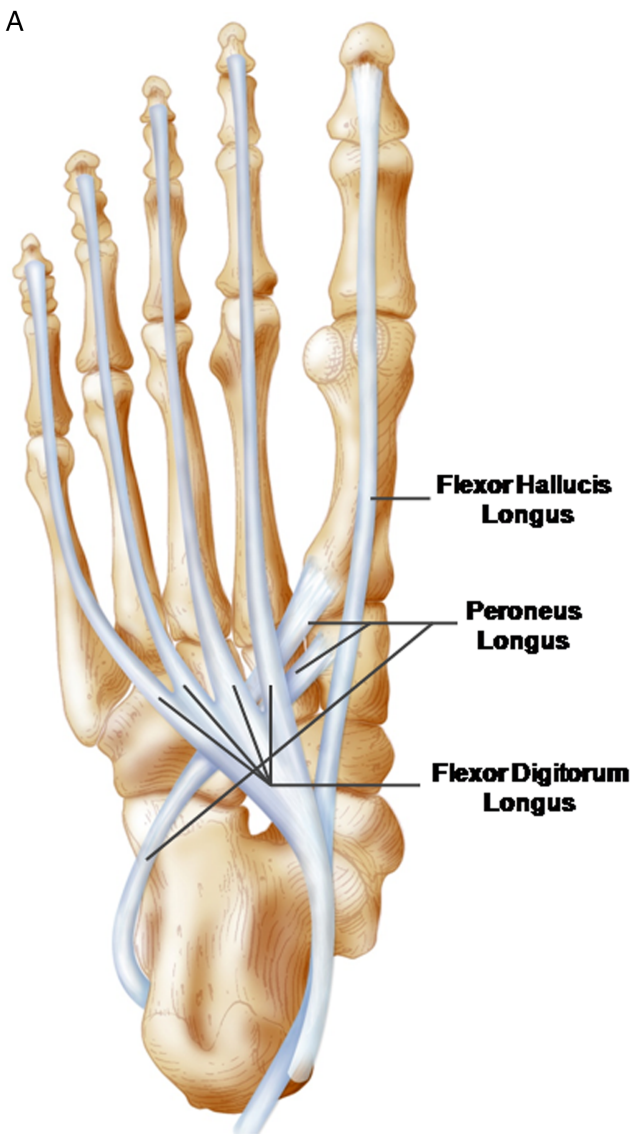

B

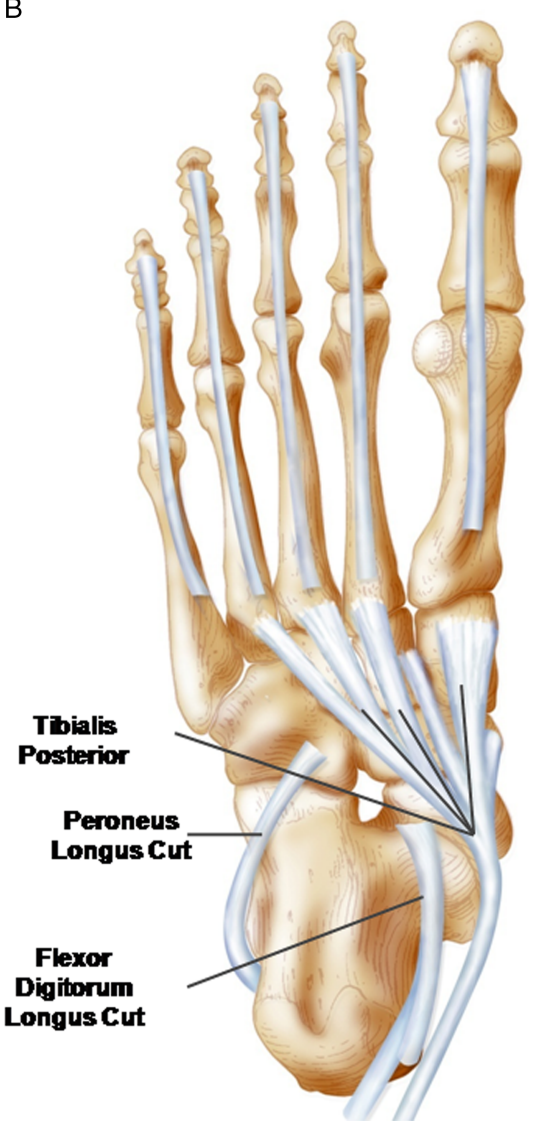

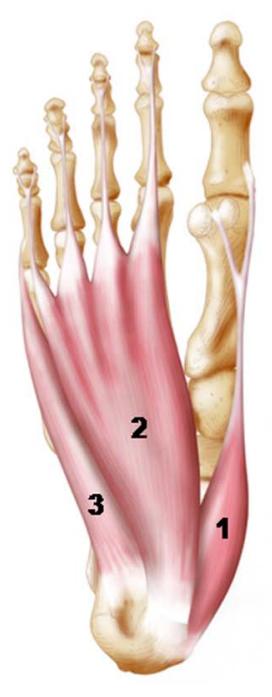
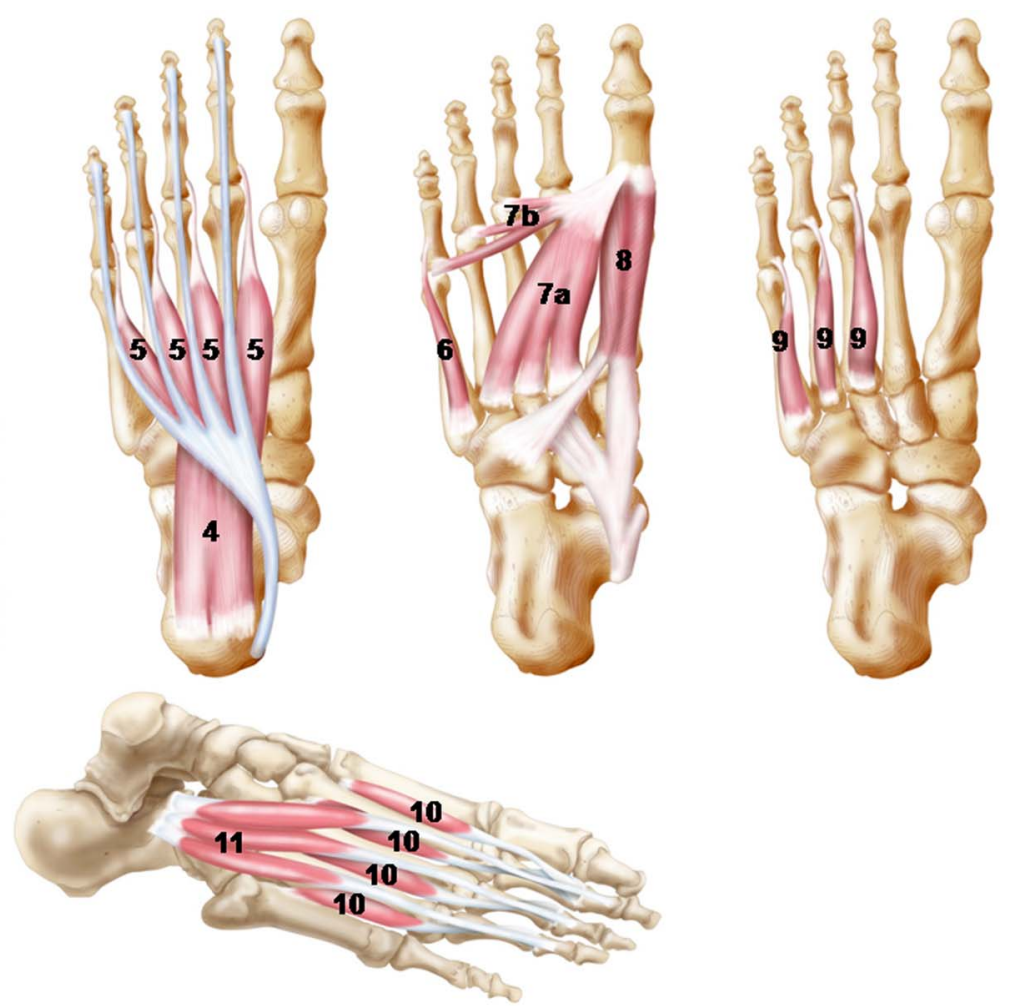

Figure 6 The intrinsic foot muscles are presented in their anatomic orientation within the four plantar layers and the dorsal intrinsic muscle. The numbers correspond to the muscles as follows: (1) abductor hallucis, (2) flexor digitorum brevis, (3) abductor digiti minimi, (4) quadratus plantae (note its insertion into the flexor digitorum tendon), (5) lumbricals (note their origin from the flexor digitorum longus tendon), (6) flexor digiti minimi, (7) adductor hallucis oblique (a) and transverse (b) heads, (8) flexor hallucis brevis, (9) plantar interossei, (10) dorsal interossei and (11) extensor digitorum brevis. 
Table 1 Functional qualities of the intrinsic foot muscles and their corresponding evidence-based descriptions

\begin{tabular}{ll}
\hline Functional quality & Description \\
\hline $\begin{array}{l}\text { Supportive of the foot } \\
\text { arches }\end{array}$ & $\begin{array}{l}\text { Diminished function of the intrinsic foot muscles leads to deleterious alterations in foot posture } \\
\text { enhances foot posture } 35\end{array}$ \\
$\begin{array}{l}\text { Activity dependent } \\
\text { Intrinsic foot muscles are more active in dynamic activities such as walking compared to standing }\end{array}$ \\
$\begin{array}{l}\text { Load dependent } \\
\text { Synergistic }\end{array}$ & $\begin{array}{l}\text { As postural demands increase, such as from double to single limb stance, so does the activity of the intrinsic foot muscles } \\
\text { The intrinsic foot muscles work together as a unit to provide dynamic arch support during the propulsive phase of gait }\end{array}$ \\
Modulating & The intrinsic foot muscles support the foot in its role as a platform for standing and lever for propelling the body during dynamic activities ${ }^{28}$
\end{tabular}

ground and is asked to maintain the foot position in single limb stance for $30 \mathrm{~s}$. The clinician observes for gross changes in navicular height and overactivity of the extrinsic muscles. ${ }^{27}$ Preliminary evidence suggests that the intrinsic foot muscle test can detect improvements in foot core function after rehabilitation in patients with lower extremity injuries, ${ }^{34} 35$ however further development of the clinimetric properties of this test is needed.

Both surface and fine wire EMG are methods of testing intrinsic foot muscle function, although these have primarily been in laboratory rather than clinical settings. Surface EMG testing has focused on the abductor hallucis, the most superficial intrinsic muscle of the medial longitudinal arch. ${ }^{32}{ }^{33}$ While EMG crosstalk is typically a concern with muscles in proximity to each other, this concern is diminished in this case because the abductor hallucis EMG activity is seen as a surrogate for all of the medially located intrinsic foot muscles as a whole. Fine wire EMG testing of the intrinsic foot muscles is ideally performed by using real-time ultrasound imaging to guide and confirm the location of the indwelling electrode. Kelly et $a l^{36}$ reported the ability to assess the activation of the abductor hallucis, flexor digitorum brevis, dorsal interossei and quadratus plantae with these methods. At this time, there is a lack of clinical studies that have used either surface or fine wire EMG to assess plantar intrinsic muscle function in lower extremity injured patients.

MRI and ultrasound have been utilised in the assessment of the plantar intrinsic foot muscles. MRI has primarily been used to assess either the cross-sectional area or the total volume of specific muscles. For example, Chang et al ${ }^{50}$ demonstrated that patients with unilateral plantar fasciitis had less total volume of the plantar intrinsic muscles in their forefoot region compared to their contralateral healthy limbs. Serial MRI examinations have been used to demonstrate more rapid atrophy of plantar intrinsic muscles in patients with diabetes with neuropathy compared to patients with diabetes without neuropathy and healthy controls. ${ }^{51}$ Similar muscle volume deficits have been identified with ultrasonography. ${ }^{48}$ As with surface EMG, the superficial location of the abductor hallucis muscle has made this muscle the primary target for ultrasound measures of cross-sectional area. ${ }^{52}$ Further research is needed to determine if ultrasound of the plantar intrinsic muscles can be used as a biofeedback tool during rehabilitation to allow patients to visualise contraction of specific muscles similar to what has been used with the lateral abdominal muscles. ${ }^{53}$

\section{FOOT CORE TRAINING}

Therapeutic exercise of the plantar intrinsic foot muscles has been traditionally described as occurring during toe flexion exercises such as towel curls and marble pick-ups. While these exercises certainly do activate some of the plantar intrinsic muscles, they also involve substantial activation of the flexor hallucis longus and flexor digitorum longus muscles. Recently, the 'short foot exercise' has been described as a means to isolate contraction of the plantar intrinsic muscles ${ }^{45} 54$ (figure 7). The foot is 'shortened' by using the intrinsic muscles to pull the first metatarsophalangeal joint towards the calcaneus as the medial longitudinal arch is elevated. As the arch raises during this exercise, it is also referred to as 'foot doming. ${ }^{56}$

We advocate Hodges' concept ${ }^{26}$ of establishing control of intrinsic foot muscle function before increasing capacity. The short foot exercise can be viewed as a foundational exercise for foot and ankle rehabilitation similar to how the abdominal drawing in manoeuvre (ADIM) is foundational to lumbopelvic core stability exercise programmes. With the ADIM, emphasis is placed on the patient learning to sense pelvic neutral and being able to contract the local stabiliser muscles to draw in the umbilicus. Care is taken to not allow activation of any global mover muscles while executing the ADIM. With the short foot exercise, emphasis should be placed on the patient learning to sense subtalar neutral with the calcaneus and the metatarsal heads on the ground and the toes neither flexed nor extended (the positioning described earlier with the intrinsic foot muscle test) and then being able to shorten the foot by using the plantar intrinsic muscles. EMG activity of the abductor hallucis, flexor digitorum brevis and quadratus plantae have been shown to increase substantially with increasing postural demand. ${ }^{36}$ Activation of the abductor hallucis has been shown to be over four times greater during short foot exercise compared to towel curl exercises in sitting and unipedal standing. ${ }^{57}$ The short foot exercise can be performed in progression from sitting to bipedal, to unipedal positions, followed by functional activities such as squats and single leg hops.

There is increasing evidence to suggest that training the foot core via short foot exercise progressions can improve foot function. For example, 4 weeks of short foot exercise training in healthy individuals reduces arch collapse as assessed by measures of navicular drop and arch height index, and improve balance ability. ${ }^{35}$ In another study, healthy individuals who completed 4 weeks of short foot exercises demonstrated improved dynamic balance compared to those who performed 4 weeks of towel curl exercises. ${ }^{58}$ However, postural control gains following a 4-week balance training home exercise programme were equivalent between healthy training groups that did and did not perform the short foot positioning during their balance exercises. ${ }^{59}$ In healthy young adults with pes planus, there were significant increases in great toe flexion strength and the cross-sectional area of the abductor hallucis muscle after 4 weeks of short foot exercises and foot orthotic intervention compared to foot orthotic intervention alone. ${ }^{57}$ Preliminary evidence demonstrates improved self-reported function in chronic ankle instability patients who performed 4 weeks of short foot 
Figure 7 The short foot manoeuvre is depicted. Note in the relaxed foot (left) the resting length of the foot (top image with solid black line). In the contracted position (right), note the change in foot length (dashed line) due to the short foot contraction drawing in the foot (arrows) from the relaxed condition (solid black line).
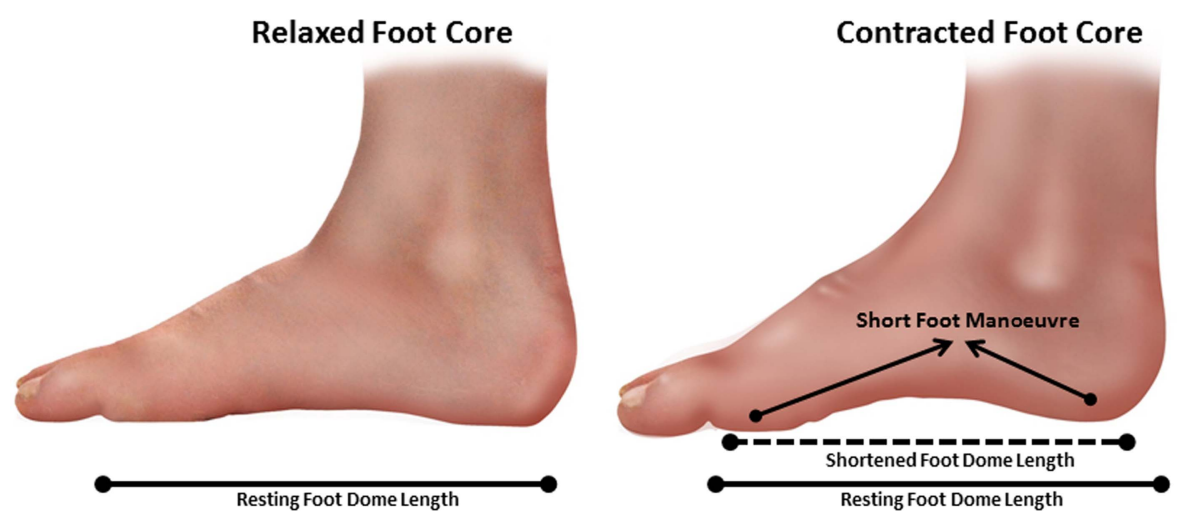

positioning during balance exercises compared to a group that did not perform the short foot positioning during their balance exercises. ${ }^{60}$ The training programmes used in these studies form a framework for functional improvements through foot core rehabilitation. There are other promising interventions for foot core training, which may offer strong functional benefits.

\section{Role of barefoot/minimal footwear training for the foot core}

Barefoot/minimal footwear walking and running may be used as a training tool to strengthen the foot core system. Robbins and Hanna $^{61}$ reported a significant reduction in the foot length (measured radiographically from the anterior aspect of the calcaneus to the first metatarsophalangeal joint) following 4 months of barefoot walking and running. The shortened foot is an indirect measure of foot strengthening as it indicates a raising of the arch. Muscle size has been directly correlated to muscle strength. ${ }^{62}$ Using this principle, Brüggemann et $a l^{63}$ measured the cross-sectional area of some of the core muscles of the foot in runners who trained for 5 months in shoes that lacked any support to the arch and rearfoot. They reported significant increases in the cross-sectional area of many of these muscles. Further studies are needed to determine whether strength and cross-sectional area gains of the foot core muscles lead to a reduction in running-related injuries.

Another advantage of being completely barefoot is the increase in sensory input received from the plantar surface of the foot. Sensory input has long been recognised for its importance in postural stability and dynamic gait patterns. $^{43} 6465$ In a study of single leg standing, postural stability was found to be significantly improved when standing bare feet as opposed to in thin socks. ${ }^{66}$ This suggests that the thin socks filter out important sensory input that assists us with our static stability. This sensory input appears to be important to dynamic stability as well. In a recent study of single leg landings, dynamic stability was improved when landing in the barefoot condition compared to a minimal running shoe and a traditional running shoe. ${ }^{67}$ In fact, stability progressively increased with decreasing amount of footwear support. These studies highlight the potential importance of sensory input to the function of the foot. Therefore barefoot activities, in safe environments, should assist in improving foot function. However, it should be noted that individuals without normal sensation should avoid barefoot activities.

\section{SUMMARY}

In summary, we hope we have increased the awareness of the importance of the foot core, making up our arch, to overall foot function. We have presented evolutionary evidence that the foot core system developed in response to the increased demands of load carriage and running. Admittedly, there is much we do not know about the intricacies of our foot mechanics. However, advancements in dynamic imaging such as biplanar videoradiography will further enhance our understanding of normal and abnormal foot kinematics. Clearly, a stronger foot is a healthier foot. To this end, we are suggesting a paradigm shift in the way we think about treating the foot. Current clinical guidelines include the use of foot orthotic devices for heel pain and plantar fasciitis, but lack any reference to strengthening of the foot. While temporary support may be needed during the acute phase of an injury, it should be replaced as soon as possible with a strengthening programme just as would be carried out for any other part of the body. Therefore, we believe that more attention needs to be focused on static and dynamic foot core function in rehabilitation programmes. These concepts can be extended to organised fitness programmes as well. Our feet were designed with the strength for unsupported endurance walking and running. Unfortunately, adding permanent support to the foot, as opposed to strengthening the foot core, is the current standard of care.

We would like to suggest that perhaps it is time for the Decade of the Foot. This type of attention to a largely ignored, but critical, part of our body might help to raise awareness of the amazing function of our feet and their underappreciated potential for improvement.

\section{What are the new findings?}

- The foot core system is comprised of interacting subsystems that provide relevant sensory input and functional stability for accommodating to changing demands during both static and dynamic activities. The interaction of these subsystems is very similar to the lumbopelvic core system.

- The plantar intrinsic foot muscles within the active and neural subsystems play a critical role in the foot core system as local stabilisers and direct sensors of foot deformation.

- Assessment of the foot core system can provide clinical insight into the ability of the foot to cope with changing functional demands.

- Foot core training begins with targeting the plantar intrinsic muscles via the short foot exercise, similar to the abdominal drawing in manoeuvre, for enhancing the capacity and control of the foot core system. 
Acknowledgements The authors would like to thank Tom Dolan, MS, the medical illustrator in this report. Mr Dolan is a medical illustrator and multimedia developer within the Department of Academic Technology at the University of Kentucky. He is an outstanding anatomical artist whose contributions to this review have been critical to the presentation of the foot core paradigm.

Contributors POM, JH and ID developed the concept for this manuscript. DB contributed the Evolution of the Human Foot section and Tom Dolan was the medical illustrator for figures 2-7. POM is the guarantor; however, the decision to publish was agreed on by all authors and contributors. The final version of the manuscript has been agreed on by all authors.

\section{Competing interests None.}

Provenance and peer review Not commissioned; externally peer reviewed.

\section{REFERENCES}

1 Kibler WB, Press J, Sciascia A. The role of core stability in athletic function. Sports Med 2006;36:189-98.

2 Bergmark A. Stability of the lumbar spine. A study in mechanical engineering. Acta Orthop Scand Supp/ 1989;230:1-54.

3 Leetun DT, Ireland ML, Willson JD, et al. Core stability measures as risk factors for lower extremity injury in athletes. Med Sci Sports Exerc 2004;36:926-34.

4 Ferber R, Noehren B, Hamill J, et al. Competitive female runners with a history of iliotibial band syndrome demonstrate atypical hip and knee kinematics. J Orthop Sports Phys Ther 2010;40:52-8.

5 Milner $C E$, Ferber $R$, Pollard $C D$, et al. Biomechanical factors associated with tibial stress fracture in female runners. Med Sci Sports Exerc 2006;38:323-8.

6 Noehren B, Davis I, Hamill J. ASB clinical biomechanics award winner 2006 prospective study of the biomechanical factors associated with iliotibial band syndrome. Clin Biomech (Bristol, Avon) 2007;22:951-6.

7 Noehren B, Hamill J, Davis I. Prospective evidence for a hip etiology in patellofemoral pain. Med Sci Sports Exerc 2013;45:1120-4.

8 Wearing SC, Smeathers JE, Urry SR, et al. The pathomechanics of plantar fasciitis. Sports Med 2006;36:585-611.

9 McPoil TG, Martin RL, Cornwall MW, et al. Heel pain—plantar fasciitis: clinical practice guidelines linked to the international classification of function, disability, and health from the orthopaedic section of the American Physical Therapy Association. J Orthop Sports Phys Ther 2008;38:A1-18.

10 Bowring B, Chockalingam N. A clinical guideline for the conservative management of tibialis posterior tendon dysfunction. Foot (Edinburgh, Scotland) 2009:19:211-17.

11 Winters $\mathrm{M}$, Eskes $\mathrm{M}$, Weir $\mathrm{A}$, et al. Treatment of medial tibial stress syndrome: a systematic review. Sports Med 2013:43:1315-33.

12 Brewer RB, Gregory AJ. Chronic lower leg pain in athletes: a guide for the differential diagnosis, evaluation, and treatment. Sports Health 2012;4:121-7.

13 Crompton RH, Vereecke EE, Thorpe SK. Locomotion and posture from the common hominoid ancestor to fully modern hominins, with special reference to the last common panin/hominin ancestor. J Anat 2008;212:501-43.

14 Susman RL, Stern JT Jr, Jungers WL. Arboreality and bipedality in the Hadar hominids. Folia Primatol (Basel) 1984;43:113-56.

15 Ward CV, Kimbel WH, Johanson DC. Complete fourth metatarsal and arches in the foot of Australopithecus afarensis. Science 2011:331:750-3.

16 Harcourt-Smith WE, Aiello LC. Fossils, feet and the evolution of human bipedal locomotion. J Anat 2004;204:403-16.

17 Bennett MR, Harris JW, Richmond BG, et al. Early hominin foot morphology based on 1.5-million-year-old footprints from Ileret, Kenya. Science 2009;323:1197-201.

18 Bramble DM, Lieberman DE. Endurance running and the evolution of Homo. Nature 2004;432:345-52.

19 Alexander RM. Energy-saving mechanisms in walking and running. J Exp Biol 1991;160:55-69.

20 Ker RF, Bennett MB, Bibby SR, et al. The spring in the arch of the human foot. Nature 1987;325:147-9.

21 Reeser LA, Susman RL, Stern JT Jr. Electromyographic studies of the human foot: experimental approaches to hominid evolution. Foot Ankle 1983;3:391-407.

22 Caravaggi P, Pataky T, Gunther M, et al. Dynamics of longitudinal arch support in relation to walking speed: contribution of the plantar aponeurosis. J Anat 2010;217:254-61.

23 Basmajian JV, Stecko G. The role of muscles in arch support of the foot. J Bone Joint Surg Am 1963;45:1184-90

24 Wang WJ, Crompton RH. The role of load-carrying in the evolution of modern body proportions. J Anat 2004;204:417-30.

25 Panjabi MM. The stabilizing system of the spine. Part I. Function, dysfunction, adaptation, and enhancement. J Spinal Disord 1992;5:383-9; discussion 97.

26 Hodges PW. Core stability exercise in chronic low back pain. Orthop Clin North Am 2003;34:245-54

27 Jam B. Evaluation and retraining of the intrinsic foot muscles for pain syndromes related to abnormal control of pronation. [October 10, 2013]. http://www.aptei. com/articles/pdf/IntrinsicMuscles.pdf
28 Gray H, Standring S, Ellis H, et al. Gray's anatomy : the anatomical basis of clinical practice 39th edn. New York: Elsevier Churchill Livingtone, 2005.

29 McKenzie J. The foot as a half-dome. Br Med J 1955;1:1068-9.

30 Ridola C, Palma A. Functional anatomy and imaging of the foot. Ital । Anat Embryol 2001;106:85-98.

31 Soysa A, Hiller C, Refshauge $K$, et al. Importance and challenges of measuring intrinsic foot muscle strength. J Foot Ankle Res 2012;5:29.

32 Fiolkowski $\mathrm{P}$, Brunt $\mathrm{D}$, Bishop $\mathrm{M}$, et al. Intrinsic pedal musculature support of the medial longitudinal arch: an electromyography study. J Foot Ankle Surg 2003:42:327-33.

33 Headlee DL, Leonard JL, Hart JM, et al. Fatigue of the plantar intrinsic foot muscles increases navicular drop. J Electromyogr Kinesiol 2008;18:420-5.

34 Drewes LK, Beazell J, Mullins M, et al. Four weeks of short foot exercises affect lower extremity function, but not alignment, in patients with lower extremity injuries. J Athl Train 2008;43:\$105.

35 Mulligan EP, Cook PG. Effect of plantar intrinsic muscle training on medial longitudinal arch morphology and dynamic function. Man Ther 2013; 18:425-30

36 Kelly $L A$, Kuitunen $S$, Racinais $S$, et al. Recruitment of the plantar intrinsic foot muscles with increasing postural demand. Clin Biomech (Bristol, Avon) 2012;27:46-51.

37 Mann R, Inman VT. Phasic activity of intrinsic muscles of the foot. J Bone Joint Surg Am 1964;46:469-81.

38 Mann RA, Hagy JL. The function of the toes in walking, jogging and running. Clin Orthop Relat Res 1979(142):24-9.

39 Cheung JT, Zhang M, An KN. Effect of Achilles tendon loading on plantar fascia tension in the standing foot. Clin Biomech 2006;21:194-203.

40 Eils $\mathrm{E}$, Nolte $\mathrm{S}$, Tewes $\mathrm{M}$, et al. Modified pressure distribution patterns in walking following reduction of plantar sensation. J Biomech 2002:35:1307-13.

41 Hoch MC, McKeon PO, Andreatta RD. Plantar vibrotactile detection deficits in adults with chronic ankle instability. Med Sci Sports Exerc 2012;44:666-72.

42 Kavounoudias A, Roll R, Roll JP. The plantar sole is a 'dynamometric map' for human balance control. Neuroreport 1998;9:3247-52.

43 Kavounoudias A, Roll R, Roll JP. Foot sole and ankle muscle inputs contribute jointly to human erect posture regulation. J Physiol 2001;532:869-78.

44 McKeon PO, Hertel J. Diminished plantar cutaneous sensation and postural control. Percept Motor Skills 2007;104:56-66.

45 Janda V, Vavrova M, Hervenova A, et al. Sensory motor stimulation. In: Liebenson C. ed Rehabilitation of the spine: a practitioner's manual. 2nd edn. Lippincott Williams \& Wilkins, 2006.

46 Hiemstra LA, Lo IK, Fowler PJ. Effect of fatigue on knee proprioception: implications for dynamic stabilization. J Orthop Sports Phys Ther 2001;31:598-605.

47 Boucher JA, Abboud J, Descarreaux M. The influence of acute back muscle fatigue and fatigue recovery on trunk sensorimotor control. J Manipulative Physiol Ther 2012;35:662-8.

48 Severinsen K, Obel A, Jakobsen J, et al. Atrophy of foot muscles in diabetic patients can be detected with ultrasonography. Diabetes Care 2007;30:3053-7.

49 Bus SA, Maas M, Michels RP, et al. Role of intrinsic muscle atrophy in the etiology of claw toe deformity in diabetic neuropathy may not be as straightforward as widely believed. Diabetes Care 2009;32:1063-7.

50 Chang R, Kent-Braun JA, Hamill J. Use of MRI for volume estimation of tibialis posterior and plantar intrinsic foot muscles in healthy and chronic plantar fasciitis limbs. Clin Biomech (Bristol, Avon) 2012;27:500-5.

51 Andreassen CS, Jakobsen J, Ringgaard S, et al. Accelerated atrophy of lower leg and foot muscles-a follow-up study of long-term diabetic polyneuropathy using magnetic resonance imaging (MRI). Diabetologia 2009;52:1182-91.

52 Cameron AF, Rome K, Hing WA. Ultrasound evaluation of the abductor hallucis muscle: reliability study. J Foot Ankle Res 2008;1:12.

53 Teyhen DS, Gill NW, Whittaker JL, et al. Rehabilitative ultrasound imaging of the abdominal muscles. J Orthop Sports Phys Ther 2007;37:450-66.

54 Greenman P. Exercise principles and prescription. In: Butler J. ed Principles of manual medicine. 2nd edn. Baltimore: Williams \& Wilkins, 1996:449-525.

55 Sauer LD, Beazell J, Hertel J. Considering the intrinsic foot musculature in evaluation and rehabilitation for lower extremity injuries. Athl Train Sports Health Care 2011;3:43-7.

56 Black M. Pilates for Feet: Pilates-Pro.com; [10 October 2013]. http://www. pilates-pro.com/pilates-pro/2009/3/24/pilates-for-feet.html

57 Jung DY, Koh EK, Kwon OY. Effect of foot orthoses and short-foot exercise on the cross-sectional area of the abductor hallucis muscle in subjects with pes planus: a randomized controlled trial. J Back Musculoskelet Rehabil 2011; 24:225-31.

58 Lynn SK, Padilla RA, Tsang KK. Differences in static- and dynamic-balance task performance after 4 weeks of intrinsic-foot-muscle training: the short-foot exercise versus the towel-curl exercise. I Sport Rehabil 2012;21:327-33.

59 Rothermel $\mathrm{S}$, Hale $\mathrm{S}$, Hertel J, et al. Effect of active foot positioning on the outcome of a balance training program. Phys Ther Sport 2004:5:98-103.

60 Sauer LD, Saliba SA, Ingersoll CD, et al. Effects of rehabilitation incorporating short foot exercises on self-reported function, static and dynamic balance in chronic ankle instability patients. J Athl Train 2010;45:S67. 
61 Robbins SE, Hanna AM. Running-related injury prevention through barefoot adaptations. Med Sci Sports Exerc 1987;19:148-56.

62 An KN, Linscheid RL, Brand PW. Correlation of physiological cross-sectional areas of muscle and tendon. J Hand Surg (Edinburgh, Scotland) 1991;16:66-7.

63 Brüggemann $G$, Potthast W, Braunstein B, et al. Effect of increased mechanical stimuli on foot muscles functional capacity. American Society of Biomechanics Annual Meeting. Cleveland, OH, 2005. http://www.asbweb.org/conferences/2005/ pdf/0553.pdf (accessed 10 Oct 2013).

64 Maurer C, Mergner T, Bolha B, et al. Human balance control during cutaneous stimulation of the plantar soles. Neurosci Lett 2001;302:45-8.
65 Meyer PF, Oddsson LI, De Luca CJ. The role of plantar cutaneous sensation in unperturbed stance. Exp Brain Res 2004;156:505-12.

66 Shinohara J, Gribble P. Five-toed socks decrease static postural control among healthy individuals as measured with time-to-boundary analysis. 2009 American Society of Biomechanics Annual Meeting. State College, PA, 2009. http://www.asbweb.org/conferences/2009/952.pdf (accessed 10 Oct 2013).

67 Rose W, Bowser B, McGrath R, et al. Effect of footwear on balance. American Society of Biomechanics Annual Meeting. Long Beach, CA, 2011. http://www. asbweb.org/conferences/2011/pdf/344.pdf (accessed 10 Oct 2013). 